Article

\title{
Critical Gap Comparison between One-, Two-, and Three-Lane Roundabouts in Qatar
}

\author{
Khaled Shaaban ${ }^{1, *(\mathbb{D})}$ and Hassan Hamad ${ }^{2}$ \\ 1 Department of Civil Engineering/Qatar Transportation and Traffic Safety Center, Qatar University, \\ Doha 2713, Qatar \\ 2 Dept. of Civil, Geological, and Environmental Engineering, University of Saskatchewan, \\ Saskatoon, SK S7N 5A9, Canada; hah093@usask.ca \\ * Correspondence: kshaaban@qu.edu.qa
}

Received: 4 April 2020; Accepted: 7 May 2020; Published: 21 May 2020

check for updates

\begin{abstract}
A critical gap value of a roundabout is mainly affected by the interactions between the circulating vehicles and vehicles entering the roundabout. These interactions are impacted by many factors, including the number of circulating lanes, the aggressiveness of local drivers, vehicle types, and the number of approaching lanes. Therefore, it is essential to locally investigate critical gap values before conducting any studies to improve capacity and delay at roundabouts. The purpose of this study is to measure and compare the critical gap values for different types of roundabouts in Qatar. More than 10,000 measurements were collected. The results showed that the critical gap values were $2.24 \mathrm{~s}, 2.55 \mathrm{~s}$, and $2.40 \mathrm{~s}$ for the one-, two-, and three-lane roundabouts, respectively. These values are also quite low when compared to values calculated in other countries, which can be an indication of driver aggressiveness and risky behavior. The study is one of the first efforts to compare three types of roundabouts and to understand the difference in operation between them in this region. The results of this study can help engineers, planners, and public agencies to plan, study, and design similar facilities.
\end{abstract}

Keywords: gap acceptance; single-lane roundabout; human factors; driver behavior

\section{Introduction}

A roundabout is an unsignalized intersection characterized by a central island and yield-on-entry approaches. Vehicles entering the circulation lanes navigate around the central island in one direction. Circulating vehicles have priority, and vehicles entering from any approach must find a gap to proceed to the circulation lanes. A gap is described as a difference in time between the tail of one circulating vehicle (gap vehicle) and the head of the following gap vehicle in the circulating lanes of a roundabout. Roundabouts are popular in many countries in Europe and Asia. In many situations, they are considered more efficient when compared to signalized intersections due to the simplicity of operation [1,2]. In addition, they also offer a safer advantage over other types of intersections due to lower speed and fewer numbers of conflicts $[3,4]$.

Capacity analysis of roundabouts is important for transportation engineers. According to the Highway Capacity Manual (HCM), in order to conduct this type of analysis, several parameters are needed. One of these parameters is the critical gap [5]. This parameter is defined as the minimum gap size (in seconds) that an entering driver would use to enter the roundabout. Typically, the entering vehicles (decision vehicles) stop to identify proper gaps before proceeding. These vehicles are more likely to reject gaps that are smaller than the critical gap value and accept gaps that are larger than it. In general, the critical gap value is an essential factor in designing or improving roundabouts. It is also an essential parameter in the modeling and simulation of roundabouts. 
The critical gap is dependent on the drivers' aggressiveness behavior, which differs from one region to another. Therefore, it is essential to have locally calibrated critical gap values. The purpose of this study is to measure and compare the critical gap values for one-, two-, and three-lane roundabouts using case studies from Qatar, and to compare the obtained values with values from other research efforts. The study considers factors such as the different types of vehicular interactions and vehicle types. This study is one of the first efforts to understand the operation of roundabouts in this region.

A few studies have attempted to compare the values of the critical gap for different types of roundabouts, including one-lane, two-lane, and modern roundabouts [6-8]. However, to our knowledge, there have been no studies that compared the critical gap values for one-, two-, and three-lane roundabouts. It should also be noted that the values established by this study for the three-lane roundabouts are not comparable since there are no similar studies on this type of roundabout in this region.

\section{Literature Review}

One of the most reliable and easy-to-use methods of estimating the critical gap was introduced by Raff and Hart in the late 1940s [9]. Based on Raff's method, the value of the critical gap $\left(t_{c}\right)$ is graphically determined by identifying the point of intercept between the two functions; $1-F\left(t_{r}\right)$ and $F\left(t_{a}\right)$. The values of $t_{r}$ and $t_{a}$ are the rejected and accepted gap times, respectively [10,11]. The original definition was set for the critical lag value, yet, the same concept applies to determine the critical gap value. This concept was adopted and applied to the critical gap estimation in several studies [12-14].

Some studies aimed to define other critical gap estimation techniques, such as the HCM, the maximum likelihood method, and Ashworth's method $[5,15,16]$. A few studies also compared the different critical gap estimation techniques. Guo [16] used various methods, such as the maximum likelihood method, Raff's method, and Ashworth's, to identify the values of the critical gap at roundabouts during rush hour. Troutbeck [15] has investigated the ability of the maximum likelihood method and the probability equilibrium method to estimate the mean and standard deviation of the critical gap. Çalişkanelli et al. [13] investigated the applicability of Raff's method and Ashworth's in Turkey. Both methods were found to provide acceptable results. However, it was recommended to modify the existing methods to improve the results by taking the local conditions into consideration.

Other studies aimed to measure the effect of different factors on the critical gap values. Different studies found that the value of the critical gap depends on many factors such as study location, time of day (peak or slack times), type of movement, queue waiting time, driver waiting time at the yield line, vehicle classification, point of departure in the circulating lanes, demographics of the driver, and availability of a passenger next to the driver [9,17]. Polus et al. [2] investigated the impact of the waiting time for the decision vehicle on the critical gap value and the effect of a newly calculated critical gap value on the capacity of a modern roundabout. The results showed that the newly calculated capacity was more than that the capacity calculated based on the HCM. The authors indicated that the lower HCM value was due to not taking into consideration the driver's behavior in reaction to extended waiting times in the calculation of the critical gap of a roundabout. Fitzpatrick et al. [18] completed a gap acceptance study on a roundabout at the University of Massachusetts campus. The results showed that the average circulating flow speed is one of the main factors affecting the critical gap values. Dahl and Lee [19] developed an empirical estimation of the capacity of roundabouts under the effect of some external factors on the value of the critical gap. They suggested the use of an adjustment factor related to trucks in the derivation of the value of the critical gap.

\section{Methods}

\subsection{Case Study}

This study was conducted in Qatar. Qatar is one of six countries located within the Arabian Gulf region, namely Bahrain, Kuwait, Oman, Saudi Arabia, and the United Arab Emirates. According 
to previous studies, driver behavior in this region is considered aggressive [20-24]. In this study, three types of roundabouts were investigated; one-, two-, and three-lane roundabouts and data were collected at four busy urban roundabouts in Qatar.

The one-lane roundabout is located in the Al Muntazah area, at the intersection of Wadi Rasheeda Street (north-south) and Hiteen Street (east-west). This roundabout has a wide circulation lane that is useful for larger trucks to navigate through the roundabout. The two-lane roundabout is located in the Wakra municipality south of Doha, at the intersection of Al Jamiyah Street (north-south) and Al Wukair Road (east-west). This roundabout represents the geometry and operation conditions of a typical two-lane roundabout in Qatar.

Since there are no similar studies on three-lane roundabouts in this region, two roundabouts were investigated. The first three-lane roundabout is located in the Al Gharrafa municipality west of Doha, at the intersection between $\mathrm{Al} \mathrm{Gharrafa} \mathrm{Road} \mathrm{(north-south)} \mathrm{and} \mathrm{Al} \mathrm{Maszhabiya} \mathrm{Street} \mathrm{(east-west);} \mathrm{it} \mathrm{is}$ called Eid Bin Mohammed roundabout. The second three-lane roundabout is located in the Al Rayyan municipality west of Doha, at the intersection between Mesaimeer Road (east-west) and Haloul Street (north-south); this roundabout is called Haloul roundabout. The two selected roundabouts are very similar in geometry, and they represent the typical configuration of three-lane roundabouts in the city of Doha.

The four roundabouts are typical roundabouts that can be found in local areas in the city of Doha, and it would not fall into any special case or category when looking into the majority of roundabouts in Doha. The roundabouts were selected to have traffic volumes to ensure a large sample size that can result in accurate values in the analysis stage. The traffic control at all sites was yield control.

\subsection{Data Collection and Extraction}

There are different methods to investigate drivers' behavior at roundabouts, including simulation [25,26], field observations [27,28], or a combination of field observations and simulation $[29,30]$. In this study, field observations were collected using video recording to obtain the necessary data for analysis. Videos were recorded during normal weekdays when high-traffic volumes could be observed. Two cameras at different angles were used at each intersection to observe the movements, interactions, and queues at the roundabouts, as shown in Figure 1. The cameras were attached to existing light poles at a 15-foot height using a telescopic pole to enable a wider view, avoid being visible by the drivers, and avoid any obstruction of heavy vehicles. Fifteen hours of video data were captured at each location. Table 1 summarizes the parameters of each site.

Video analysis software was used to analyze the videos frame by frame in order to obtain the required data for further analysis. Rejected and accepted gaps' data were extracted from the recorded videos. A gap, whether accepted or rejected, must satisfy two conditions to be counted as a data point in this study. First, the decision vehicle should come to a full stop at the yield line of the approach. Second, the circulation lane(s) corresponding to an approach lane that is occupied by a decision vehicle has (have) to be occupied by at least two vehicles that form a gap.

A gap is accepted if a driver stops entirely at the roundabout, then proceeds once a gap becomes available in the circulating flow. Moreover, a gap is rejected when the driver does not move from the approach when a gap is available inside the roundabout, irrespective of its size. The gap value is measured as the difference in time (seconds) between the rear end of the first gap vehicle, and the front end of the following gap vehicle, measured at the same reference line extending from the nose of the splitter island at the entry of the approach pointing to the center of the roundabout. This line should be perpendicular to the circulation lanes to ensure consistent measuring of gaps across the circulation lanes.

The data extraction revealed much higher traffic volumes at the multilane roundabouts compared to the one-lane roundabout. In addition, pedestrian and bicycle use was light at the roundabout sites. This is common to this region, as confirmed by previous studies in Qatar [31-33]. Moreover, because of 
the relatively large circulating volumes, most drivers had to stop and then perform the gap acceptance process at the roundabouts.

Table 1. Geometry and Traffic Characteristics of the Roundabout Sites Observed.

\begin{tabular}{|c|c|c|c|c|c|c|c|}
\hline Site & $\begin{array}{l}\text { Number of } \\
\text { Circulatory } \\
\text { Lanes }\end{array}$ & Location & $\begin{array}{c}\text { Inscribed } \\
\text { Circle } \\
\text { Diameter (m) }\end{array}$ & $\begin{array}{l}\text { Approach } \\
\text { Observed }\end{array}$ & $\begin{array}{l}\text { Type of } \\
\text { Day }\end{array}$ & Time & $\begin{array}{l}\text { Length of } \\
\text { Video } \\
\text { (hr) }\end{array}$ \\
\hline 1 & 1 & $\begin{array}{l}\text { Rawadat Al } \\
\text { Khail } \\
\text { St./Hiteen St. }\end{array}$ & 32.2 & Westbound & \multirow{4}{*}{ Weekday } & \multirow{4}{*}{$\begin{array}{l}\text { 6:00 a.m. } \\
\quad \text { to } \\
\text { 9:00 p.m. }\end{array}$} & \multirow{4}{*}{15} \\
\hline 2 & 2 & $\begin{array}{l}\text { Al Jamiyah } \\
\text { St./Al Wukair } \\
\text { Rd. }\end{array}$ & 68.9 & Eastbound & & & \\
\hline 3 & 3 & $\begin{array}{l}\text { Al Gharrafa } \\
\text { Rd./Al } \\
\text { Maszhabiliya } \\
\text { St. }\end{array}$ & 85.9 & Northbound & & & \\
\hline 4 & 3 & $\begin{array}{c}\text { Mesaimeer } \\
\text { Rd./Haloul St. }\end{array}$ & 83.3 & Westbound & & & \\
\hline
\end{tabular}
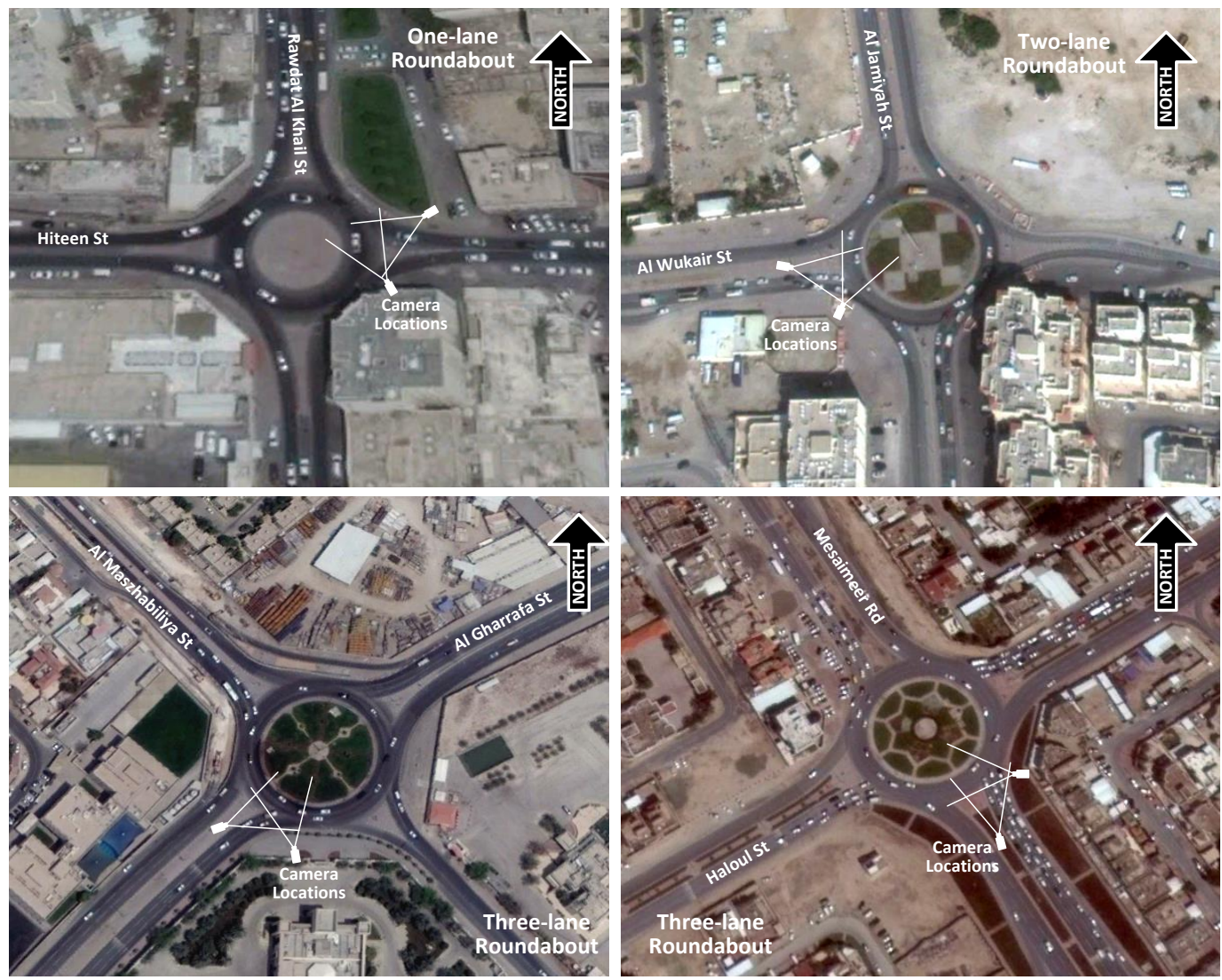

Figure 1. Aerial Photos Showing the Roundabouts and Cameras' Locations.

\subsection{Vehicular Types and Interaction Cases}

In this study, vehicles were classified into three categories; passenger cars, medium-sized vehicles, and heavy vehicles. The passenger car category includes sedans, four-wheel drive, and vans. The medium vehicle category involves single-unit trucks, minibuses, and ambulances. The heavy vehicle category covers all types of heavy vehicles. Typically, vehicular interactions are defined as the interactions (action-reaction) between at least two vehicles at any given time and space. Vehicle interactions differ based on the size of the roundabout. As the number of lanes increases, the complexity of the vehicular interactions increases. 


\subsubsection{One-Lane Roundabout}

The one-lane roundabout has only one interaction case, where the decision vehicle interacts with the gap vehicles in the roundabout circulation lane, as shown in Figure 2.

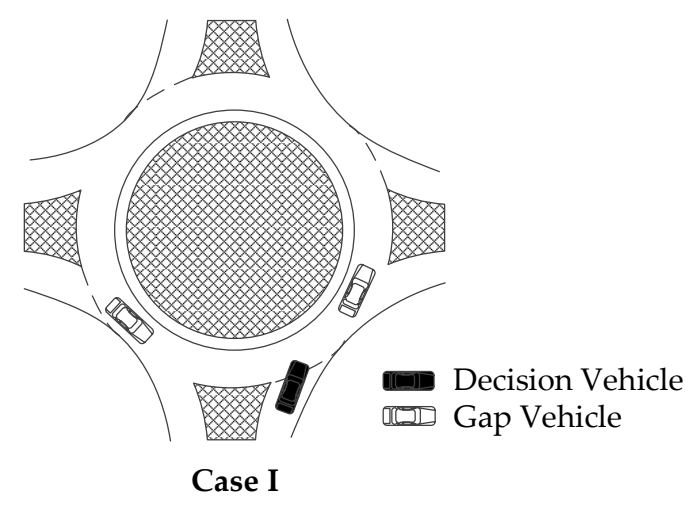

Figure 2. Interaction Case for a One-Lane Roundabout.

\subsubsection{Two-Lane Roundabout}

In the case of a two-lane roundabout, there are five interaction cases (see Figure 3). Cases 1 and 2 take place when the gap vehicles are located in the far circulation lane or in the near circulation lane, respectively, and the decision vehicle is located in the inside lane. Cases 4 and 5 take place when one gap vehicle is located in the far circulation lane, and the other is located in the near circulation lane. Case 3 occurs when both gap vehicles are located in the near circulation lane, and the decision vehicle is located in the outside lane. Lags were not considered in the analysis to get more accurate values for the critical gaps and avoid large gaps. Furthermore, the case when the gap vehicles are located in the far circulation lane, and the decision vehicle is located in the outside lane, was not included in the analysis since the decision vehicle does not have to interact with any gap vehicles.

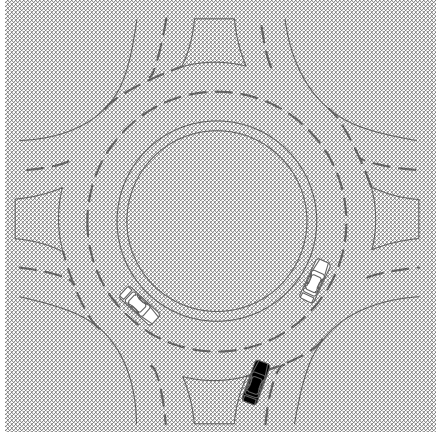

Case II-1

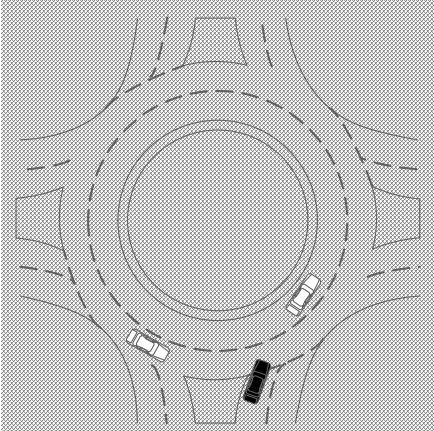

Case II-4

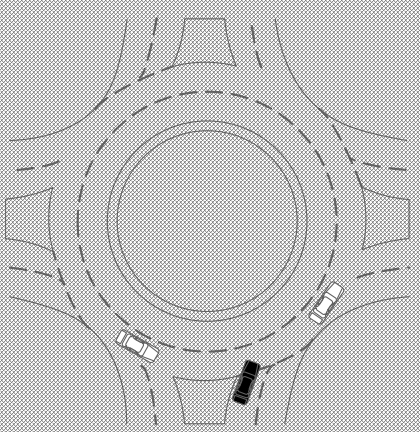

Case II-2

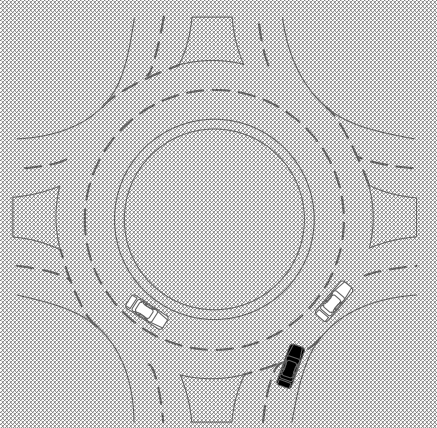

Case II-5

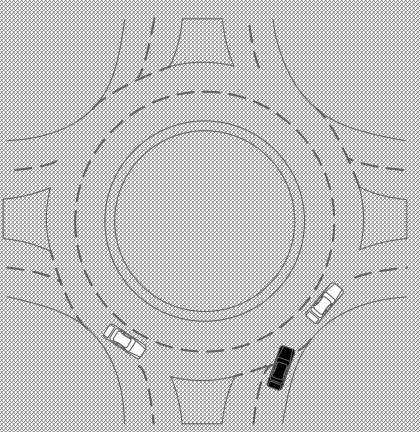

Case II-3

Decision Vehicle Gap Vehicle

Figure 3. Interaction Cases for a Two-Lane Roundabout. 


\subsubsection{Three-Lane Roundabouts}

After observing the decision vehicles in the case of three-lane roundabouts, it was found that some vehicles (follow vehicles) accept the gaps in groups. In this case, the follow vehicles wait for a decision vehicle to proceed then move simultaneously at the same time. As illustrated in Figure 4, seven cases were observed ( 1 to 7 ). Cases 1, 2, and 3 occur when the decision vehicle, in the inside lane, accepts a gap, and the follow vehicles, in the middle and outside lanes, take advantage and use the same gap. From a data efficiency point of view, the critical gap for the follow vehicles was not included into the analysis in these cases. Case 4 occurs when a similar situation occurs between a decision vehicle in the middle lane, and a follow vehicle in the outside lane. Cases 5, 6, and 7 involve only one decision vehicle that accepts an individual gap. More information about this concept is available in the previous work of the authors [34].

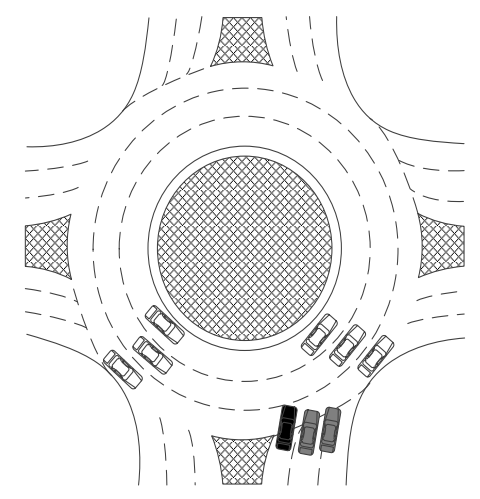

Case III-1

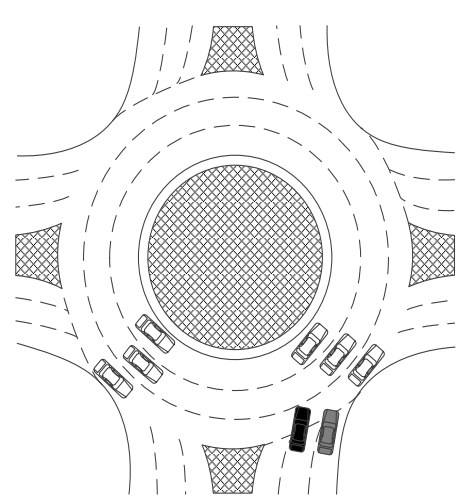

Case III-4

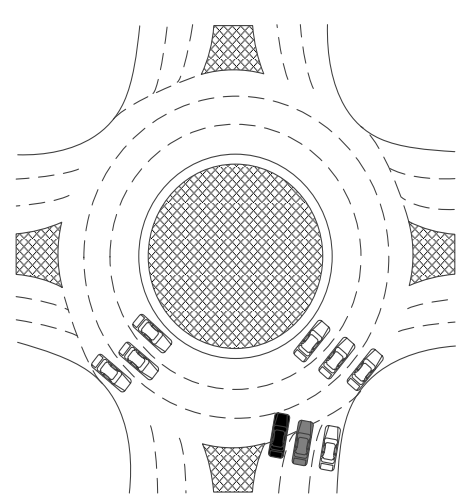

Case III-2

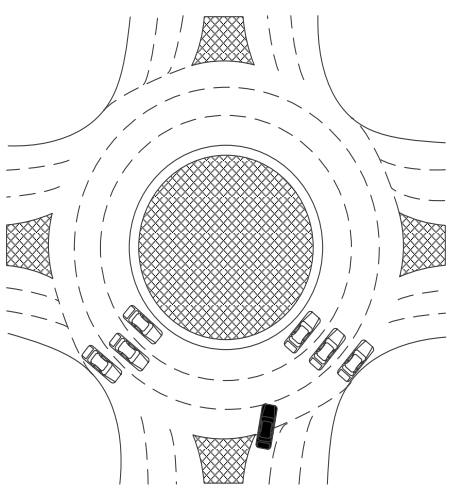

Case III-5

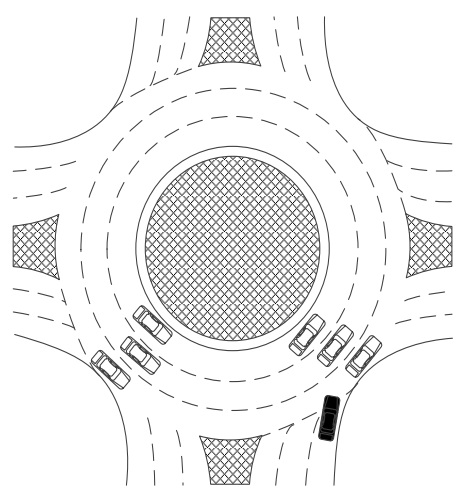

Case III-7

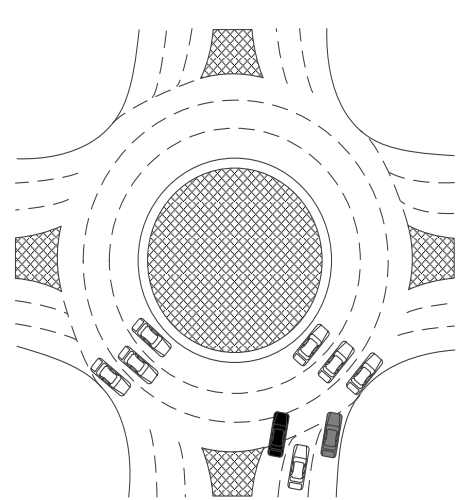

Case III-3

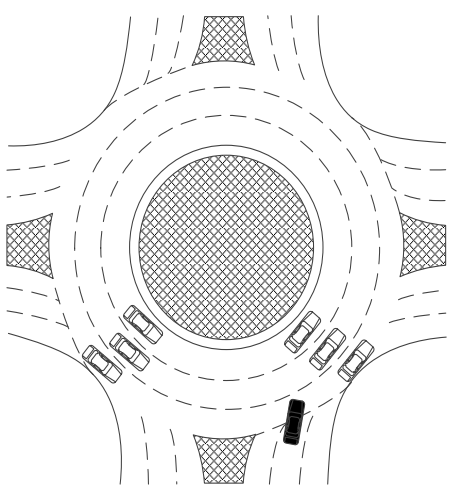

Case III-6

- Decision Vehicle

Follow Vehicle

Gap Vehicle

Figure 4. Interaction Cases for a Three-Lane Roundabout. 


\section{Analysis}

The analysis was conducted using Raff's method since it is one of the favorable methods used in estimating the critical gap. The method is easy to use and produces reasonably accurate results [14]. According to Raff's method, the critical gap is obtained by finding the point of intersection between the cumulative distribution functions (CDFs) of the rejected and accepted gaps. This value can be obtained either graphically or by equalizing the sigmoidal functions of the cumulative distribution curves. In this study, the graphical method was applied to the overall data for each type of roundabout in addition to the different interaction cases and vehicle types.

\subsection{One-Lane Roundabout}

The number of observations at the on-lane roundabout was over 1500 gaps, and there is only one case of vehicular interactions. The overall critical gap was found to be $2.24 \mathrm{~s}$, as shown in Table 2 . The CDFs that yielded this value can be seen in Figure 5. More than $90 \%$ of observed vehicles were passenger cars; $6 \%$ were medium vehicles, and $1.9 \%$ were heavy vehicles. The critical gap values for three types of vehicles, passenger car, medium vehicle, and heavy vehicle, were obtained (see Figure 5). The passenger cars had the lowest critical gap value (2.24 s), followed by the medium vehicles $(2.27 \mathrm{~s})$ and the heavy vehicles $(2.93 \mathrm{~s})$, as shown in Table 2.

Table 2. Critical Gap Values for the One-Lane Roundabout.

\begin{tabular}{ccccc}
\hline Classification & Type & Sample Size & Percentage & $\begin{array}{c}\text { Critical Gap } \\
\text { (s) }\end{array}$ \\
\hline \multirow{2}{*}{ Gap Acceptance } & Accepted & 461 & $29.4 \%$ & 2.24 \\
& Rejected & 1106 & $70.6 \%$ & \\
\hline Vehicle Type & Passenger Car & 1443 & $92.1 \%$ & 2.24 \\
& Medium Vehicle & 94 & $6.0 \%$ & 2.27 \\
& Heavy Vehicle & 30 & $1.9 \%$ & 2.93 \\
\hline Total & & 1567 & $100.0 \%$ & \\
\hline
\end{tabular}

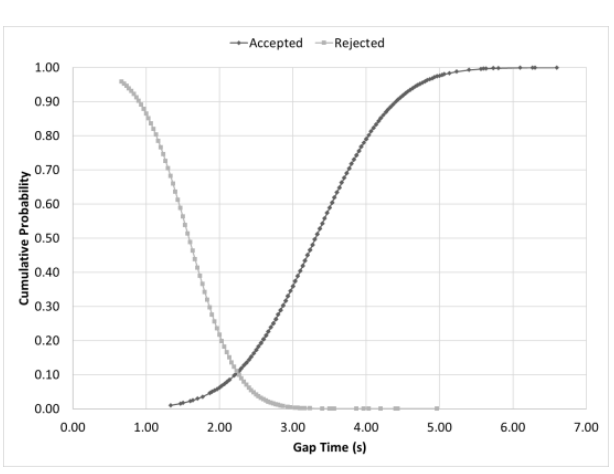

Overall Critical Gap

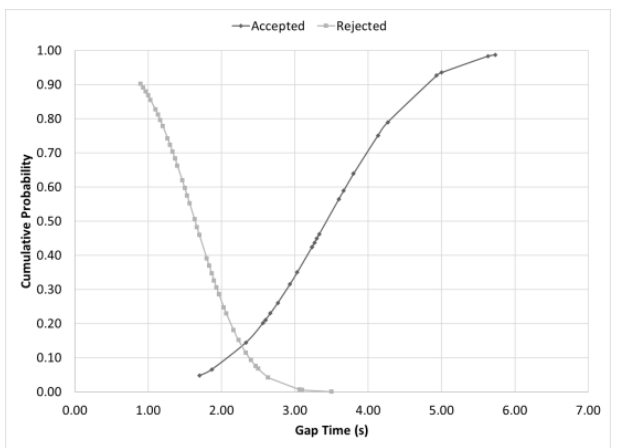

Critical Gap of Medium Vehicles

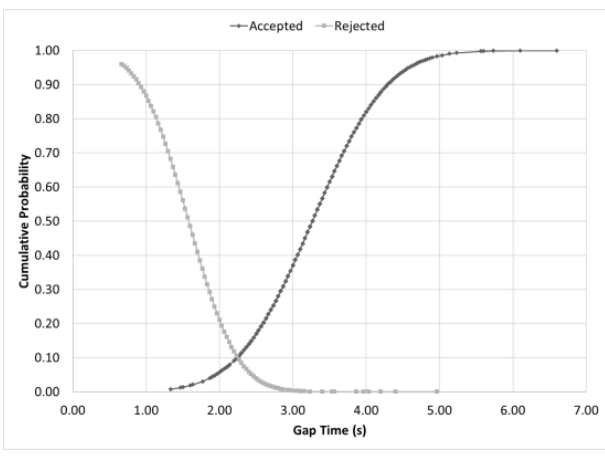

Critical Gap of Passenger Cars

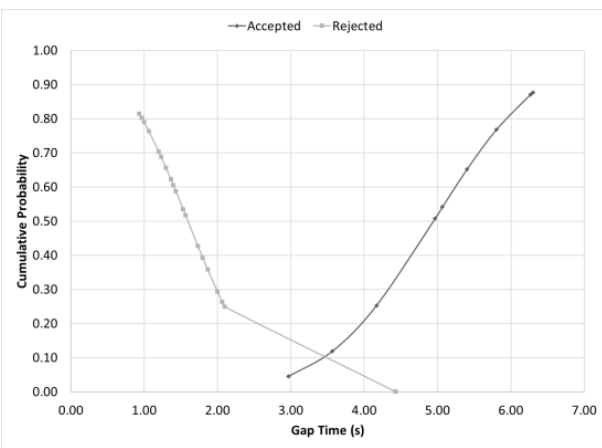

Critical Gap of Heavy Vehicles

Figure 5. CDFs for the One-Lane Roundabout. 


\subsection{Two-Lane Roundabout}

The number of observations at the two-lane roundabout was over 4000 gaps. The overall critical gap was found to be $2.55 \mathrm{~s}$, as shown in Table 3. The CDFs that yielded this value can be seen in Figure 6. Most of the interactions (66.1\%) involved vehicles of case 1 and yielded a critical gap of $2.51 \mathrm{~s}$. The minimum critical gap was $2.33 \mathrm{~s}$ (case 4), and the maximum critical gap was $2.87 \mathrm{~s}$ (case 5). More than $95 \%$ of observed vehicles were passenger cars, $3.6 \%$ were medium vehicles, and $0.9 \%$ were heavy vehicles (see Table 3). The passenger cars had the lowest critical gap value ( $2.5 \mathrm{~s})$, followed by the medium vehicles (2.9 s) and the heavy vehicles (2.95 s), as shown in Figure 6.

Table 3. Critical Gap Values for the Two-Lane Roundabout.

\begin{tabular}{ccccc}
\hline Classification & Type & Sample Size & Percentage & $\begin{array}{c}\text { Critical Gap } \\
\text { (s) }\end{array}$ \\
\hline \multirow{2}{*}{ Gap Acceptance } & Accepted & 1468 & $34.3 \%$ & 2.55 \\
& Rejected & 2806 & $65.7 \%$ & \\
\hline Case & 1 & 2824 & $66.1 \%$ & 2.51 \\
& 2 & 286 & $6.7 \%$ & 2.50 \\
& 3 & 24 & $0.6 \%$ & 2.95 \\
& 4 & 551 & $12.9 \%$ & 2.33 \\
Vehicle Type & 5 & 4089 & $13.8 \%$ & 2.87 \\
& Passenger Car & 153 & $95.5 \%$ & 2.50 \\
& Medium Vehicle & 40 & $3.6 \%$ & 2.90 \\
\hline \multirow{2}{*}{ Total } & Heavy Vehicle & 4274 & $100.9 \%$ & 2.95 \\
\hline
\end{tabular}

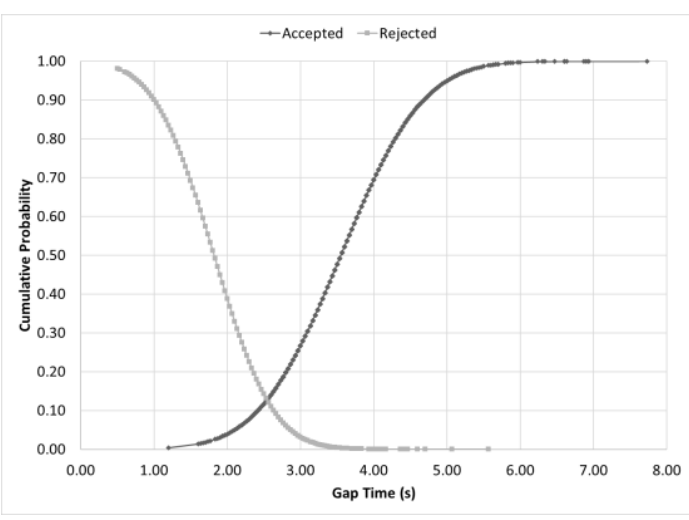

Overall Critical Gap

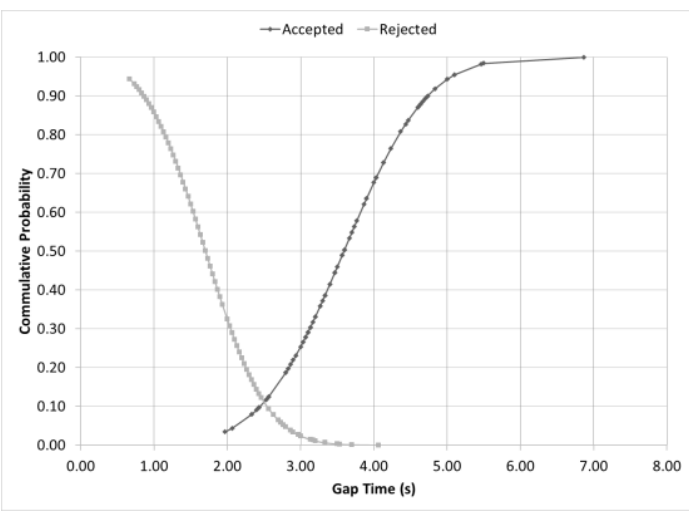

Critical Gap of Case 2

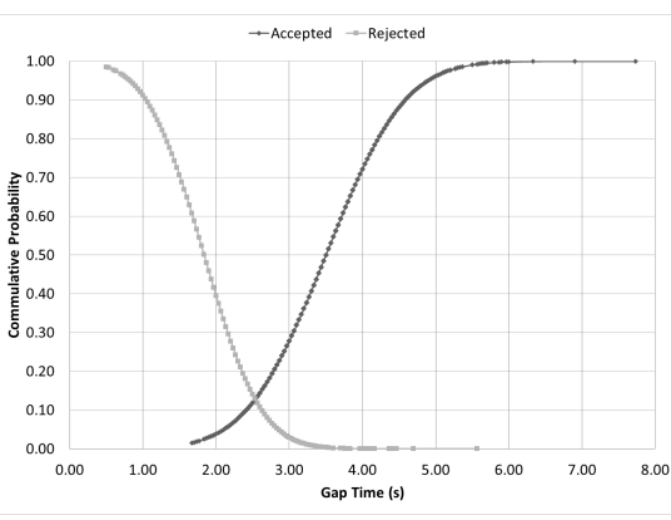

Critical Gap of Case 1

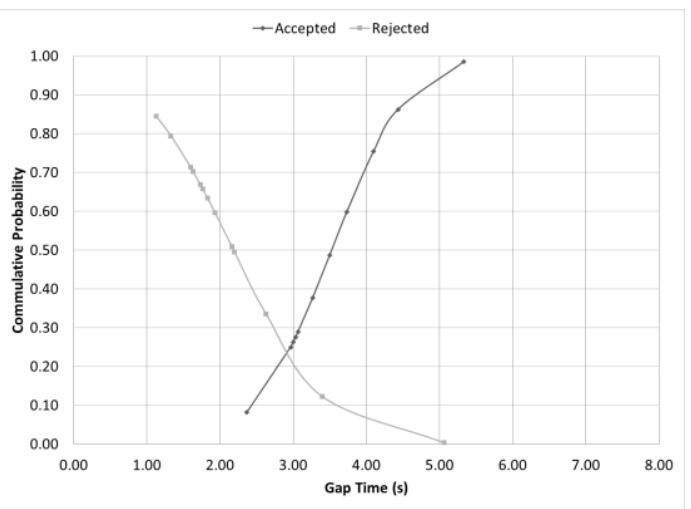

Critical Gap of Case 3

Figure 6. Cont. 


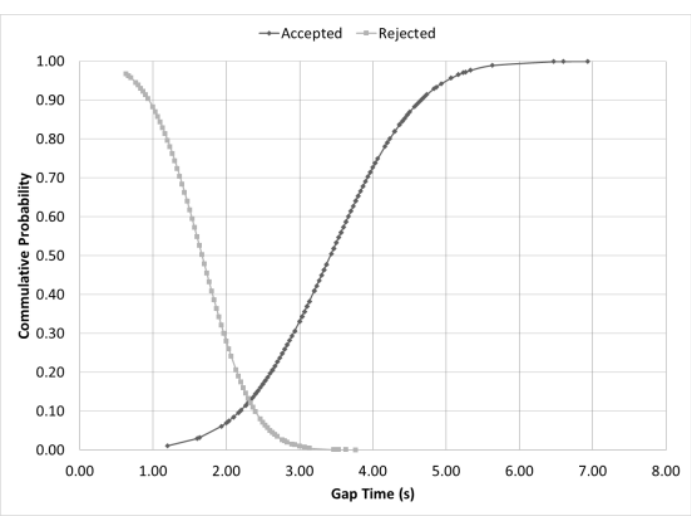

Critical Gap of Case 4

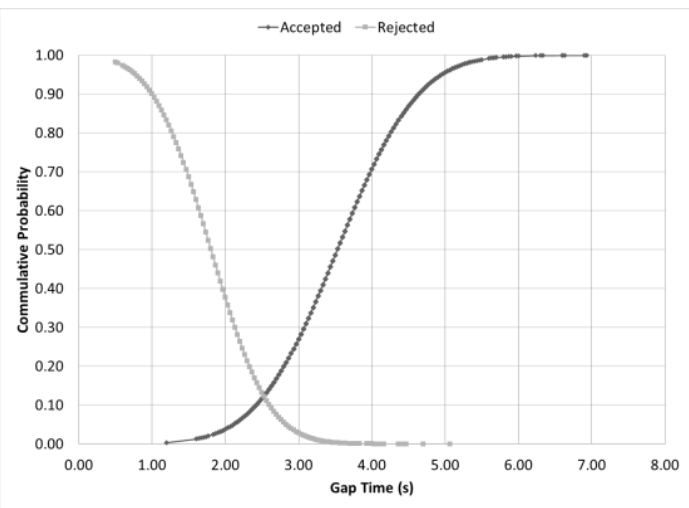

Critical gap of Passenger Vehicles

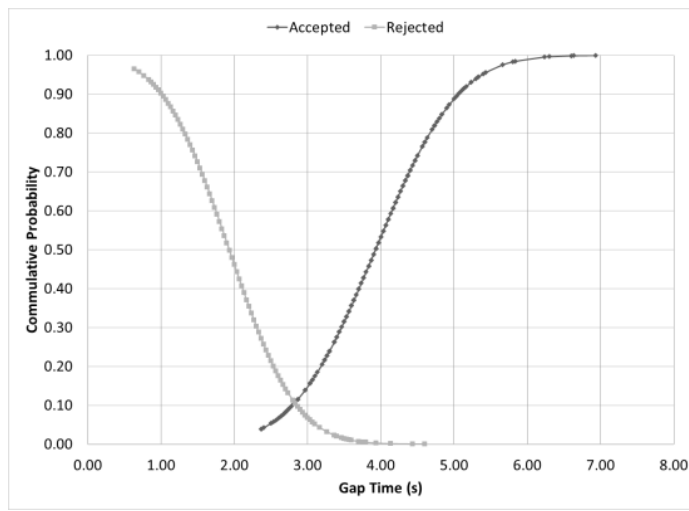

Critical Gap of Case 5

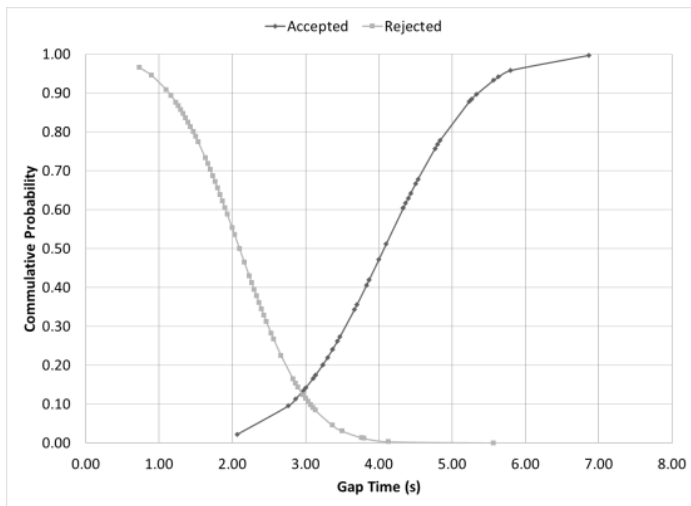

Critical Gap of Medium Vehicles

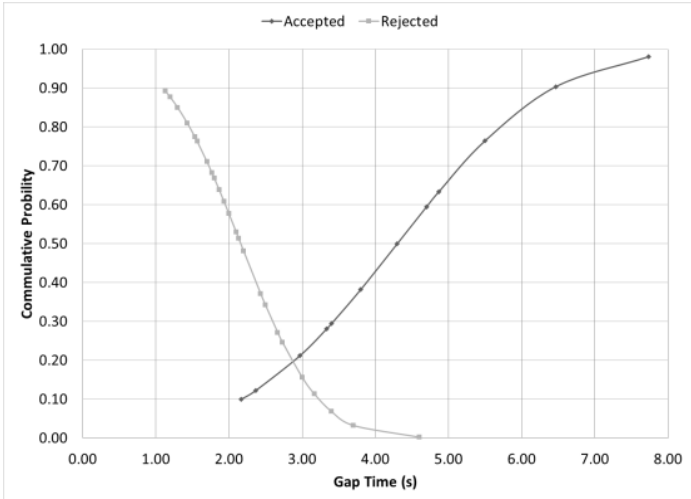

Critical Gap of Heavy Vehicles

Figure 6. CDFs for the Two-Lane Roundabout.

\subsection{Three-Lane Roundabout}

The number of observations at the three-lane roundabouts exceeded 4500 . The overall critical gap was found to be $2.40 \mathrm{~s}$, as shown in Table 4 . The CDFs that yielded this value can be seen in Figure 7. Most of the interactions (75\%) involved vehicles of case 1 and yielded a critical gap of $2.45 \mathrm{~s}$. The minimum critical gap was $2.11 \mathrm{~s} \mathrm{(case} \mathrm{5),} \mathrm{and} \mathrm{the} \mathrm{maximum} \mathrm{critical} \mathrm{gap} \mathrm{was} 2.47 \mathrm{~s}$ (case 4). More than $95 \%$ of observed vehicles were passenger cars; $2.7 \%$ were medium vehicles, and $1.9 \%$ were heavy vehicles. The passenger cars had the lowest critical gap value $(2.39 \mathrm{~s})$, followed by the medium vehicles (2.53 s) and the heavy vehicles (3.03 s), as shown in Table 4. 
Table 4. Critical Gap Values for the Three-Lane Roundabouts.

\begin{tabular}{ccccc}
\hline Classification & Type & Sample Size & Percentage & $\begin{array}{c}\text { Critical Gap } \\
\text { (s) }\end{array}$ \\
\hline \multirow{2}{*}{ Gap Acceptance } & Accepted & 2137 & $45.6 \%$ & 2.40 \\
& Rejected & 2547 & $54.4 \%$ & 2.45 \\
Case & 1 & 3512 & $75.0 \%$ & 2.22 \\
& 2 & 420 & $9.0 \%$ & 2.33 \\
& 3 & 385 & $8.2 \%$ & 2.47 \\
& 4 & 27 & $0.6 \%$ & 2.11 \\
& 5 & 326 & $7.0 \%$ & Not enough data \\
& 7 & 3 & $0.2 \%$ & Not enough data \\
\hline \multirow{2}{*}{ Vehicle Type } & Passenger Car & 4472 & $0.1 \%$ & 2.39 \\
& Medium Vehicle & 125 & $95.5 \%$ & 3.03 \\
\hline Total & Heavy Vehicle & 87 & $2.7 \%$ & \\
\hline
\end{tabular}

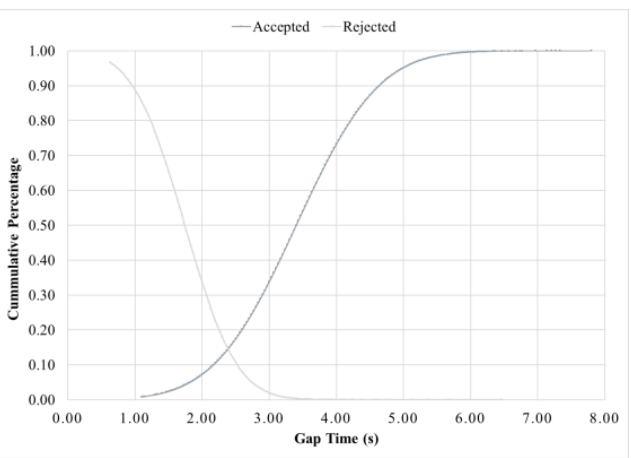

Overall Critical Gap

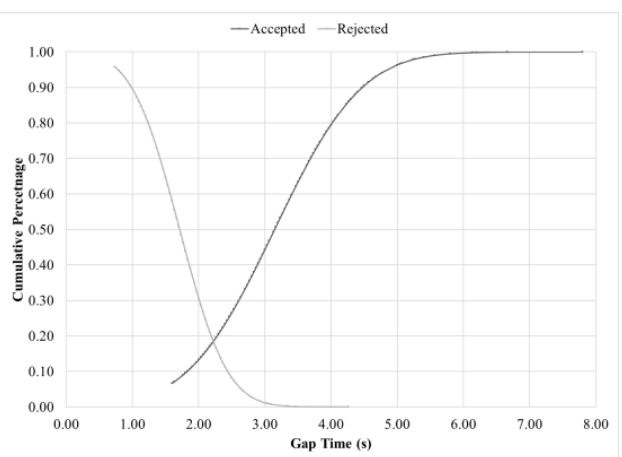

Critical Gap for Case 2

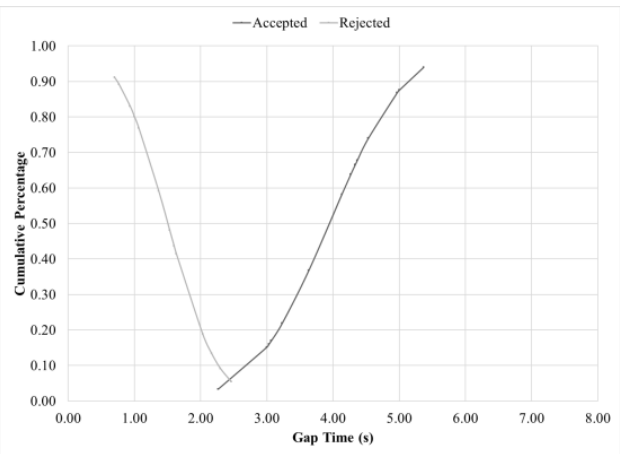

Critical Gap for Case 4

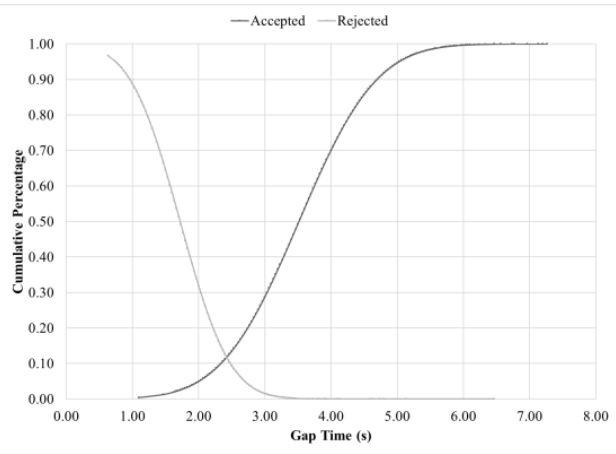

Critical Gap for Case 1

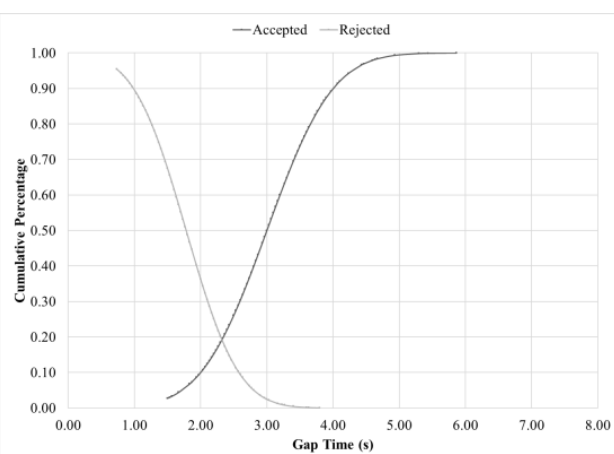

Critical Gap for Case 3

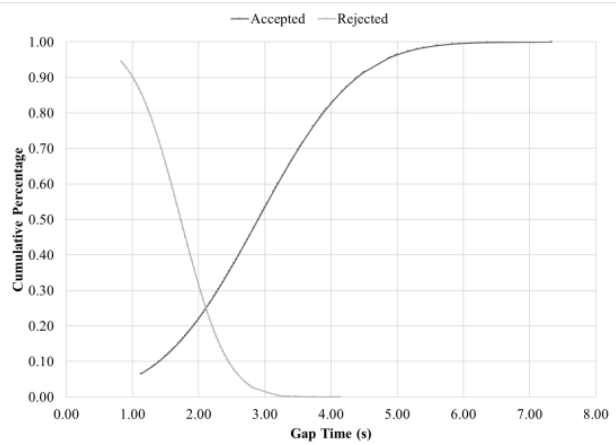

Critical Gap for Case 5

Figure 7. Cont. 


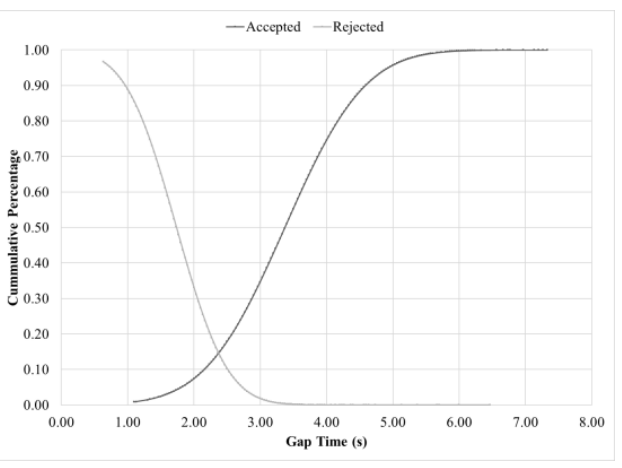

Critical Gap for Passenger Vehicles

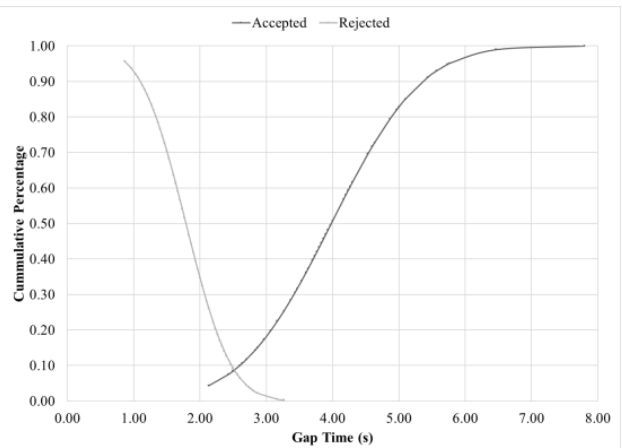

Critical Gap for Medium Vehicles

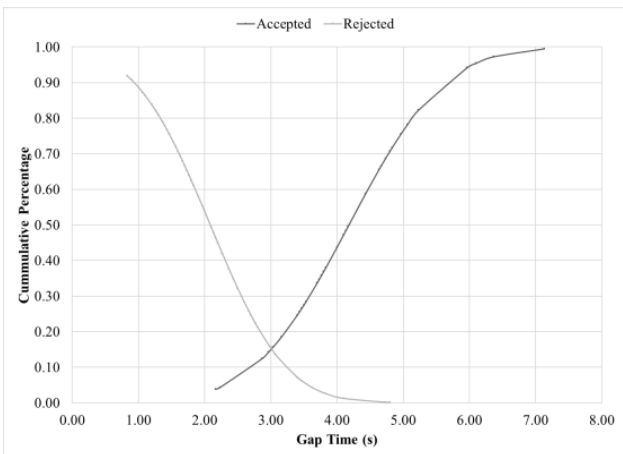

Critical Gap for Heavy Vehicles

Figure 7. CDFs for the Three-Lane Roundabouts.

\section{Results and Discussion}

This study presents a detailed estimation of the critical gap for three types of roundabouts in Qatar. Data were collected from one-, two-, and three-lane roundabouts with different layouts. More than 10,000 interactions were recorded. The data were classified based on vehicle types and interaction cases, and the analysis was performed using Raff's method. For the one-lane roundabout, the overall critical gap value was found to be $2.24 \mathrm{~s}$. Most of the interaction cases involved passenger cars constituting more than $92 \%$ of the collected data points. The critical gap of the passenger cars was $2.24 \mathrm{~s}$, compared to $2.27 \mathrm{~s}$ and $2.93 \mathrm{~s}$ for the medium and heavy vehicles, respectively.

For the two-lane roundabout, the overall critical gap value was found to be $2.55 \mathrm{~s}$. There was a significant difference between the critical gap values for the different interaction cases; case 4 had the lowest critical gap value of $2.33 \mathrm{~s}$, and case 3 and 5 had a critical gap value of $2.95 \mathrm{~s}$ and 2.87, respectively. There was also a difference between the critical gap values corresponding to different vehicle types. The passenger cars had a critical gap value of $2.50 \mathrm{~s}$, compared to $2.90 \mathrm{~s}$ and $2.95 \mathrm{~s}$ for medium and heavy vehicles, respectively.

The overall critical gap value for the three-lane roundabouts was $2.40 \mathrm{~s}$, which is lower than the two-lane roundabout. These results were unexpected as the interaction cases in three-lane roundabouts are far more complicated than that of the two-lane ones. It should be noted that the original study included only one three-lane roundabout. To verify the results, data were collected at an additional three-lane roundabout. The second roundabout data also showed a lower overall critical gap than the two-lane roundabout. These results can be explained by the group gap acceptance behavior described in the study. Similar to the case of the one- and two-lane roundabout, the passenger cars had a critical gap value of $2.39 \mathrm{~s}$, compared to $2.53 \mathrm{~s}$ and $3.03 \mathrm{~s}$ for medium and heavy vehicles, respectively. Similar results were found in studies that compared the critical gap value for different vehicle types [35].

Furthermore, the critical gap values were compared to values obtained from previous studies, as shown in Table 5. The results seem to differ significantly (lower) from the results obtained in other 
countries/regions. These results can be an indication of the driver aggressiveness behavior in this region, which, in turn, can affect the capacity of roundabouts.

Table 5. Summary of Past Roundabout Related Studies.

\begin{tabular}{ccc}
\hline Method $^{*}$ & Critical Gap (s) & Location \\
\hline One-Lane Roundabout & & \\
\hline Maximum Likelihood Method & 3.94 & Florida and Maryland, USA [36] \\
Maximum Likelihood Method & 4.80 & California, USA [28] \\
Raff's Method & 2.55 & Maryland, USA [37] \\
Raff's Method & 2.20 & Massachusetts, USA [18] \\
\hline Two-Lane Roundabout & & \\
\hline Maximum Likelihood Method & L $4.70 / R ~ 4.40$ & California, USA [28] \\
Maximum Likelihood Method & 3.58 & Stockholm, Sweden [3] \\
Raff's Method & 2.91 & Dalian, China [16] \\
Raff's Method & 3.53 & Simulation [12] \\
\hline
\end{tabular}

* No values were available for three-lane roundabouts.

A few limitations with the current study should be noted. The study did not consider cyclists' and pedestrians' impact on the gap values at all locations due to their limited number in Qatar in general. Moreover, the number of observations for some of the cases was limited (30 or less). Additional data should be collected in the future to verify the results for these cases. Furthermore, the effect of adjacent traffic control devices such as signalized intersections or interchanges was not accounted for as well. The vehicular interaction cases that were investigated in this study were the legal interaction cases. All the illegal cases, such as a decision vehicle in the outside lane entering the roundabout in the far circulation lane, were eliminated. Such cases do not resemble a proper gap acceptance maneuver, and they were considered careless or high-risk maneuvers. Furthermore, the study did not consider roundabouts with slip lanes. These special cases should be investigated as part of future studies.

Finally, the outcomes of this study are thought to reflect, more accurately, the behavior of the drivers in Qatar and this region. The obtained values can be utilized in different applications such as designing new roundabouts, generating capacity models, and assessing the level of service of existing roundabouts instead of the currently used values in the international standards. They can also be used as an input in the capacity or simulation models. Moreover, the methodology can be used to conduct similar studies in other locations with different conditions.

Author Contributions: Conceptualization and research framework: K.S.S.; methodology: K.S.S. and H.H.; data collection: K.S.S. and H.H.; writing-original draft preparation: K.S.S. and H.H.; writing—review and editing: K.S.S. and H.H. All authors have read and agreed to the published version of the manuscript.

Funding: This publication was made possible by an NPRP award [NPRP 4-1170-2-456] from the Qatar Research Fund (a member of the Qatar Foundation). The statements made herein are solely the responsibility of the author.

Conflicts of Interest: The authors declare no conflict of interest.

\section{References}

1. Polus, A.; Shmueli, S. Analysis and Evaluation of the Capacity of Roundabouts. Transp. Res. Rec. 1997, 1572, 99-104. [CrossRef]

2. Polus, A.; Lazar, S.S.; Livneh, M. Critical gap as a function of waiting time in determining roundabout capacity. J. Transp. Eng. 2003, 129, 504-509. [CrossRef]

3. Kusuma, A.; Koutsopoulos, H.N. Critical Gap Analysis of Dual Lane Roundabouts. Procedia-Soc. Behav. Sci. 2011, 16, 709-717. [CrossRef]

4. Sisiopiku, V.P.; Oh, H.-U. Evaluation of Roundabout Performance Using SIDRA. J. Transp. Eng. 2001, 127, 143-150. [CrossRef] 
5. Transportation Research Board of the National Academics. HCM 2010: Highway Capacity Manual; The National Academies of Sciences, Engineering, and Medicine: Washington, DC, USA, 2010.

6. Macioszek, E. Models of critical gaps and follow-up headways for turbo roundabouts. In Proceedings of the Scientific and Technical Conference Transport Systems Theory and Practice, Katowice, Poland, 17-19 September 2018.

7. Macioszek, E. The comparison of models for critical headways estimation at roundabouts. In Contemporary Challenges of Transport Systems and Traffic Engineering; Springer: Cham, Switzerland, 2017; pp. 205-219.

8. Macioszek, E. The comparison of models for follow-up headway at roundabouts. In Proceedings of the Scientific and Technical Conference Transport Systems Theory and Practice, Katowice, Poland, 18-20 September 2017.

9. Raff, M.S.; Hart, J.W. A Volume Warrant for Urban Stop Signs; The Eno Foundation for Highway Traffic Control: Westport, CT, USA, 1950.

10. Miller, A. Nine Estimators of Gap-Acceptance Parameters; Bulletin Transport Section, Civil Engineering; Elsevier: New York, NY, USA, 1972.

11. Brilon, W.; Koenig, R.; Troutbeck, R.J. Useful estimation procedures for critical gaps. Transp. Res. Part A Policy Pract. 1999, 33, 161-186. [CrossRef]

12. Guo, R.-J.; Wang, X.-J.; Wang, W.-X. Estimation of critical gap based on Raff's definition. Comput. Intell. Neurosci. 2014, 2014, 16. [CrossRef]

13. Çalişkanelli, P.; Özuysal, M.; Tanyel, S.; Yayla, N. Comparison of different capacity models for traffic circles. Transport 2009, 24, 257-264. [CrossRef]

14. Tupper, S.; Knodler, M.A., Jr.; Fitzpatrick, C.; Hurwitz, D.S. Estimating Critical Gap-A Comparison of Methodologies Using a Robust, Real-World Data Set. In Proceedings of the Transportation Research Board 92nd Annual Meeting, Washington, DC, USA, 13-17 January 2013.

15. Troutbeck, R. Estimating the mean critical gap. Transp. Res. Rec. J. Transp. Res. Board 2014, 2461, 76-84. [CrossRef]

16. Guo, R. Estimating critical gap of roundabouts by different methods. In Proceedings of the 6th Advanced Forum on Transportation of China (AFTC 2010), Beijing, China, 16 October 2010.

17. Tupper, S.M.; Knodler, M.A., Jr.; Hurwitz, D.S. Connecting Gap Acceptance Behavior with Crash Experience. In Proceedings of the 3rd International Conference on Road Safety and Simulation, Indianapolis, Indiana, 14-16 September 2011.

18. Fitzpatrick, C.D.; Abrams, D.S.; Tang, Y.; Knodler, M.A., Jr. Spatial and temporal analysis of driver gap acceptance behavior at modern roundabouts. Transp. Res. Rec. J. Transp. Res. Board 2013, 2388, 14-20. [CrossRef]

19. Dahl, J.; Lee, C. Empirical estimation of capacity for roundabouts using adjusted gap-acceptance parameters for trucks. Transp. Res. Rec. J. Transp. Res. Board 2012, 2312, 34-45. [CrossRef]

20. Shaaban, K.; Gharraie, I.; Sacchi, E.; Kim, I. Severity analysis of red-light-running-related crashes using structural equation modeling. J. Transp. Saf. Secur. 2019, 1-20. [CrossRef]

21. Shaaban, K.; Pande, A. Evaluation of Red Light Camera Enforcement Using Traffic Violations. J. Traffic Transp. Eng. (Engl. Ed.) 2018, 5, 66-72. [CrossRef]

22. Shaaban, K.; Wood, J.S.; Gayah, V.V. Investigating driver behavior at minor-street stop-controlled intersections in Qatar. Transp. Res. Rec. J. Transp. Res. Board 2017, 2663, 109-116. [CrossRef]

23. Statistical Analysis Office, Ministry of Interior. Traffic Accidents Report for the Year 2011; Ministry of Interior: Doha, Qatar, 2012.

24. Bener, A.; Crundall, D.; Özkan, T.; Lajunen, T. Mobile phone use while driving: A major public health problem in an Arabian society, State of Qatar-Mobile phone use and the risk of motor vehicle crashes. J. Public Health 2010, 18, 123-129. [CrossRef]

25. Belz, N.P.; Aultman-Hall, L.; Montague, J. Influence of priority taking and abstaining at single-lane roundabouts using cellular automata. Transp. Res. Part C Emerg. Technol. 2016, 69, 134-149. [CrossRef]

26. Lakouari, N.; Ez-Zahraouy, H.; Benyoussef, A. Traffic flow behavior at a single lane roundabout as compared to traffic circle. Phys. Lett. A 2014, 378, 3169-3176. [CrossRef]

27. Lee, D.; You, J.; Kim, D.; Lee, S. An Analysis of Roundabout Application Effects Based on Before and After Field Studies. Int. J. Highw. Eng. 2013, 15, 111-119. [CrossRef] 
28. Xu, F.; Tian, Z. Driver behavior and gap-acceptance characteristics at roundabouts in California. Transp. Res. Rec. J. Transp. Res. Board 2008, 2071, 117-124. [CrossRef]

29. Shaaban, K.; Abou-Senna, H.; Elnashar, D.; Radwan, E. Assessing the impact of converting roundabouts to traffic signals on vehicle emissions along an urban arterial corridor in Qatar. J. Air Waste Manag. Assoc. 2019, 69, 178-191. [CrossRef]

30. Chen, X.; Lee, M.S. A case study on multi-lane roundabouts under congestion: Comparing software capacity and delay estimates with field data. J. Traffic Transp. Eng. (Engl. Ed.) 2016, 3, 154-165. [CrossRef]

31. Shaaban, K. Why Don't People Ride Bicycles in High-Income Developing Countries, and Can Bike-Sharing Be the Solution? The Case of Qatar. Sustainability 2020, 12, 1693. [CrossRef]

32. Shaaban, K. Analysis of Pedestrian Crossing Speeds at Signalized Intersections in Qatar. Arab. J. Sci. Eng. 2019, 44, 4467-4476. [CrossRef]

33. Shaaban, K. Assessing Sidewalk and Corridor Walkability in Developing Countries. Sustainability 2019, 11, 3865. [CrossRef]

34. Shaaban, K.; Hamad, H. Group Gap Acceptance: A New Method to Analyze Driver Behavior and Estimate the Critical Gap at Multilane Roundabouts. J. Adv. Transp. 2018, 2018, 1350679. [CrossRef]

35. Macioszek, E.; Kang, N. Assessment of Psychotechnical Parameters of Vehicle Drivers on Roundabout Hitachi Taga, Japan. Sci. J. Sil. Univ. Technol. Ser. Transp. 2019, 103, 93-103. [CrossRef]

36. Flannery, A.; Datta, T. Operational performance measures of American roundabouts. Transp. Res. Rec. J. Transp. Res. Board 1997, 1572, 68-75. [CrossRef]

37. Mensah, S.; Eshragh, S.; Faghri, A. A critical gap analysis of modern roundabouts. In Proceedings of the 89th Annual Meeting of the Transportation Research Board, Washington, DC, USA, 10-14 January 2010.

(C) 2020 by the authors. Licensee MDPI, Basel, Switzerland. This article is an open access article distributed under the terms and conditions of the Creative Commons Attribution (CC BY) license (http://creativecommons.org/licenses/by/4.0/). 